\title{
Screening for diabetes: hope and despair
}

\author{
P. T. Sawicki
}

Received: 12 January 2012 / Accepted: 7 March 2012 /Published online: 19 April 2012

(C) Springer-Verlag 2012

\begin{abstract}
This commentary discusses whether screening for type 2 diabetes or earlier normalisation of blood glucose levels or initiation of non-antihyperglycaemic agents or any other diabetes-specific treatment can help reduce the excess associated risks for macrovascular morbidity and mortality. The available data indicate that screening with the sole aim of decreasing the lead time between diagnosis and treatment is very unlikely to reduce these risks. In contrast to macrovascular complications, some microvascular events such as background retinopathy could theoretically be prevented by earlier diagnosis and better glycaemic control, particularly in relatively young type 2 diabetic patients. This, however, remains to be shown in controlled prospective intervention trials.
\end{abstract}

Keywords Antihyperglycaemic treatment - Cardiovascular events · Cholesterol · Complications · Diabetes · Hypertension · Mortality $\cdot$ Quality of life · Risk stratification $\cdot$ Screening

The rising prevalence of type 2 diabetes is predicted to further increase worldwide and will continue to create a considerable burden of suffering and health costs [1]. The main problem, which remains unsolved, is still the excessive risk of cardio- and cerebrovascular morbidity and mortality. Most disappointingly, broadly effective population-based diabetes prevention programmes are not in sight [2] and even very sophisticated intensified blood glucose treatment

\section{P. T. Sawicki $(\square)$}

Institute for Health Economics and Clinical Epidemiology,

Medical Faculty of the University of Cologne,

Gleuler Strasse 176-178,

Cologne, Germany

e-mail: Peter.Sawicki@uk-koeln.de strategies have not yielded satisfying results in type 2 diabetes [3]. This has prompted ideas for alternative approaches, including the hypothesis that we may be failing because we intervene too late. Indeed, the onset of type 2 diabetes may occur more than 5 years before the diagnosis [4]. In the UK Prospective Diabetes Study (UKPDS), 50\% of newly diagnosed patients with relatively good metabolic control already had diabetes-related complications [5] and in the Hoorn study the prevalence of macrovascular complications was higher in the unscreened population compared with the screened sample [6]. These data created the hope that screening for type 2 diabetes would identify diabetic patients before complications develop and subsequent intensified treatment strategies could reduce the risk [7]. But is this hope realistic?

\section{Benefits from earlier reduction of glycaemia?}

In epidemiological studies the major burden of diabetesthe excessive risk of cardio- and cerebrovascular morbidity and mortality - is already present before blood glucose concentrations reach diabetic values [8]. Despite the fact that in observational studies glycaemia is closely associated with cardiovascular risk [9], we now have convincing evidence from randomised controlled trials that, with the use of intensive glycaemic control in type 2 diabetes, including in those patients in the early stages of the disease, there is no reduction in all-cause mortality [10]. In fact, a reduction in $\mathrm{HbA}_{1 \mathrm{c}}$ of about $0.9 \%(9.8 \mathrm{mmol} / \mathrm{mol})$ over a period of 4-5 years increased the risk ratio for total mortality nonsignificantly by 1.02 (95\% CI $0.91,1.13)$, significantly reduced non-fatal myocardial infarction by around $0.24 \%$ (absolute risk reduction) but had no impact on microvascular hard endpoints $[10,11]$. 


\section{Benefits from earlier initiation of non-antihyperglycaemic treatment options?}

Screening that results in decreasing the delay between diagnosis and treatment is of course not limited to the lowering of blood glucose, but could potentially cover all effective treatment options for the reduction of cardiovascular risk. However, are there, at present, any possible interventions that are proven to be effective primarily in diabetic, but not in non-diabetic, patients at risk for cardiovascular events?

Interventions that have been proposed as relevant only for patients with diabetes include specific therapeutic target levels for blood pressure and cholesterol, as well as different classes of antihypertensive drug treatment and prescription of acetylsalicylic acid. However, a meta-analysis provided evidence that different antihypertensive regimens had comparable effects on cardiovascular events in diabetic and nondiabetic patients [12]. Furthermore, there is limited evidence that lower blood pressure goals are more effective in hypertensive diabetic, compared with non-diabetic, patients [12]. It should be noted, however, that this meta-analysis included several trials with control groups in which blood pressure was kept above the normotensive threshold of 140/ $90 \mathrm{mmHg}$, and so does not answer the question of whether normotensive patients with diabetes benefit from antihypertensive treatment. The Action to Control Cardiovascular Risk in Diabetes trial examined the benefits of lowering of systolic blood pressure to $<120$ versus lowering to $<140 \mathrm{mmHg}$ [13]. After a mean follow-up of 4.7 years the primary composite outcome (non-fatal myocardial infarction, non-fatal stroke, or death from cardiovascular causes) was not significantly different between the study groups, indicating no benefit of blood pressure lowering within the normotensive range in diabetic patients [13]. Of course, effective antihypertensive treatment should be offered to all hypertensive patients irrespective of their blood glucose concentrations.

Likewise, the effects of cholesterol lowering treatment with statins yielded similar results in diabetic and nondiabetic patients; most importantly, in both groups the beneficial effect of statins was independent of the pre-treatment blood cholesterol levels [14]. No data from randomised controlled trials are available to answer the question of whether cholesterol treatment targets should differ between diabetic and non-diabetic patients. Hence, in my view, it can be asserted on the basis of the published evidence to date that LDL-cholesterol targets need not be different in people with and without diabetes. To date, the results of acetylsalicylic acid treatment for primary prevention of cardiovascular events are, all in all, somewhat inconclusive and a recent meta-analysis could not demonstrate a clear benefit of acetylsalicylic acid for primary prevention in diabetic patients. However, this result may differ between men and women [15].

\section{Improved risk stratification}

Information regarding the presence or absence of diabetes could potentially improve patients' and doctors' treatment decisions. Patients with diabetes face a higher risk of cardiovascular complications and the number needed to treat is, in most cases, lower for diabetic patients. However, some risk calculators do not include the variable 'diabetes' [16, 17]. Furthermore, the National Institute for Health and Clinical Excellence (NICE) states that those who are 40 years of age or older and have an additional risk factor such as diabetes are automatically considered to be at high risk for cardiovascular disease and a risk calculation is not necessary [18]. Therefore, the information derived from current risk calculators is very often insufficient to support patients' decisions for or against a certain treatment. In addition, there is no evidence that treatment decisions either with or without such information would lead to improved outcomes or to higher patient or doctor satisfaction.

\section{Results from screening intervention trials}

The Anglo-Danish-Dutch Study of Intensive Treatment in People with Screen Detected Diabetes in Primary Care (ADDITION) trial investigated the effect of early multifactorial treatment after a diabetes diagnosis by screening in a population between 40 and 69 years in a cluster-randomised study design [19]. The intervention included lowering of blood glucose to an $\mathrm{HbA}_{1 \mathrm{c}}$ target below $7 \%(53 \mathrm{mmol} / \mathrm{mol})$, maintaining blood pressure $\leq 135 / 85 \mathrm{mmHg}$ and keeping total cholesterol $<5 \mathrm{mmol} / \mathrm{l}$ in patients without, and $<4.5 \mathrm{mmol} / \mathrm{l}$ in patients with, ischaemic heart disease. Furthermore, acetylsalicylic acid was added in patients receiving antihypertensive medication. In the control group, family physicians were only provided with the diagnostic test results, and then provided routine care. The treatment effects on $\mathrm{HbA}_{1 \mathrm{c}}$ yielded only small differences between the groups while blood pressure and total- and LDL-cholesterol were reduced to a greater extent, and acetylsalicylic acid was prescribed more often, in the intervention group. After a mean follow-up of 5.3 years the risk of a first cardiovascular event was not significantly reduced by an earlier intensification of treatment. It is important to bear in mind that this trial did not directly assess the effects of screening for diabetes since screening was initiated in both study groups and only the intensity of subsequent treatment differed.

In this issue of Diabetologia, Rahman and colleagues present the long-term results of a parallel group cohort 
screening intervention of the Ely cohort [20]. A random non-diabetic sample from this cohort aged 40-65 years was invited at baseline to participate in screening for diabetes and cardiovascular risk factors every 5 years. These patients were then compared with randomly selected individuals who were invited to a screening for diabetes 10 years later. In total, 199 patients with diabetes were registered, of which 116 were in the screened and 83 in the unscreened population. The median duration of diabetes was 5 years in the screened and 1.7 years in the unscreened sample. At follow-up, glycaemic control was better in the screened population $\left(\mathrm{HbA}_{1 \mathrm{c}} 7.0 \%\right.$ vs $7.4 \% ; 53.0$ vs $\left.57.4 \mathrm{mmol} / \mathrm{mol}\right)$; however, this difference was not statistically significant. In the screened patient group, non-proliferative diabetic retinopathy and peripheral neuropathy were non-significantly less common ( $21 \%$ and $34 \%$, respectively) compared with the unscreened group (35\% and 39\%, respectively). Microalbuminuria occurred in $22 \%$ vs $23 \%$ of patients and macroalbuminuria was present in four screened and one unscreened patient. Cases of stroke, myocardial infarction and ischaemic heart disease were comparable between the groups.

It is possible that this study was too small to show statistically significant effects of earlier diagnosis and treatment of the diabetes-related risk of non-proliferative diabetic retinopathy and neuropathy, but in any case these results do not support the presence of an important impact of screening on diabetic complications. In a previous report from the same study, a non-significantly lower mortality risk in the screened cohort was reported [21]. However, this was the case only in the period between 1990 and 1999 and was not replicated in the period between 2000 and 2008. Taken together, the Ely cohort screening study failed to show any long-term benefit from screening for diabetes. It is possible that screening using $\mathrm{HbA}_{1 \mathrm{c}}$ values could yield better results than using an oral glucose challenge. It would immediately identify those patients who are at the highest risk of complications and benefit most from antihyperglycaemic intervention. However, the value of this remains to be shown.

\section{Can screening be harmful?}

Screening for diabetes might not be totally harmless. The diagnosis of diabetes is not generally associated with important adverse events [22, 23]. However, short-term anxiety may increase [24]. In one trial it was found that patients who screened positive perceived their general health to be significantly poorer and exhibited a higher state of anxiety, worry and depression [25]. These effects were small but the long-term social and psychological impact resulting from the labelling of screen-detected diabetic patients has not been well evaluated. Screening negatively for diabetes does not seem to promote false reassurance [26]. However, it may detract the physician's attention from the scrupulous control of cardiovascular risk factors in non-diabetic patients, leading to sub-optimal control compared with patients who screened positive for diabetes [27]. Most importantly, the diagnosis of diabetes can also have other negative impacts on patients' life such as increased insurance premiums and difficulties with employment.

\section{Conclusions}

In summary, the conclusion that needs to be drawn is disappointing: macrovascular pathology develops long before diabetes is present and intensive blood glucose treatment does not reduce this mortality risk. So far, randomised controlled trials have not identified any treatment geared towards the reduction of cardiovascular risk that would be effective in diabetic patients only, as opposed to a nondiabetic population. Once an indication is present, earlier specific therapeutic interventions in eligible patients should be considered irrespective of the presence of diabetes, and the vast majority, if not all, effective treatment options should be considered in both diabetic and non-diabetic patients. Furthermore, earlier intensive multifactorial treatment in screen-detected diabetic patients has yielded no better results than treatment undertaken at a later point [19]. No statistically significant reduction in diabetic complications occurred in the group of screen-detected, compared with routinely detected, diabetic patients $[20,21]$. If this is true, then screening for diabetes with the sole aim of decreasing the lead time between diagnosis and treatment is very unlikely to reduce the major burden of macrovascular morbidity and mortality in type 2 diabetes. In contrast to macrovascular complications, some microvascular events such as background retinopathy could theoretically be prevented by an earlier diagnosis and better glycaemic control, particularly in relatively young type 2 diabetic patients. This, however, remains to be shown in controlled prospective intervention trials. It is probable that such a hypothetical reduction of background retinopathy can only be achieved in a small subsample of type 2 diabetic patients and may possibly reduce the need for laser-photocoagulation in a few, whereas it is unlikely that cases of blindness could be prevented.

Facing the continuously increasing number of diabetic patients, the maximal potential benefit derived from screening for diabetes is relatively small compared with what we will have to achieve in future diabetes care. Thus, instead of waiting for studies showing positive effects of overall screening for diabetes, we should initiate detection and treatment programmes to systematically identify and treat diabetic patients at risk for complications such as leg amputations, which can effectively be prevented [28]. 
Duality of interest The author declares that there is no duality of interest associated with this manuscript.

Contribution statement The author was solely responsible for the conception, drafting and final approval of this article.

\section{References}

1. Wild S, Roglic G, Green A, Sicree R, King H (2004) Global prevalence of diabetes: estimates for the year 2000 and projections for 2030. Diabetes Care 27:1047-1053

2. Davies MJ, Tringham JR, Troughton J, Khunti KK (2004) Prevention of type 2 diabetes mellitus. A review of the evidence and its application in a UK setting. Diabet Med 21:403-414

3. Yudkin JS, Richter B, Gale EAM (2010) Intensified glucose lowering in type 2 diabetes: time for a reappraisal. Diabetologia 53:2079-2085

4. Harris MI, Klein R, Welborn TA, Knuitman MW (1992) Onset of NIDDM occurs at least 4-7 yr before clinical diagnosis. Diabetes Care 15:815-819

5. UK prospective Diabetes Study 6 (1990) Complications in newly diagnosed type 2 diabetic patients and their association with different clinical and biometrical risk factors. Diabetes Res 13:1-11

6. Spijkerman AMW, Henry RMA, Dekker JM et al (2004) Prevalence of macrovascular disease amongst type 2 diabetic patients detected by targeted screening and patients newly diagnosed in general practice: the Hoorn Screening Study. J Intern Med 256:429-436

7. Echouffo-Tcheugui JB, Simmons RK, Williams KM et al (2009) The ADDITION-Cambridge trial protocol: a cluster-randomised controlled trial of screening for type 2 diabetes and intensive treatment for screen-detected patients. BMC Public Health 9:136

8. Balkau B, Pyörälä M, Shipley M et al (1998) High blood glucose concentration is a risk factor for mortality in middle-aged nondiabetic men. 20-year follow-up in the Whitehall Study, the Paris Prospective Study and the Helsinki Policemen Study. Diabetes Care 21:360-367

9. Stratton IM, Adler AI, Neil HA et al (2000) Association of glycaemia with macrovascular and microvascular complications of type 2 diabetes (UKPDS35): prospective observational study. BMJ 321:405-412

10. Hemmingsen B, Lund SS, Gluud C et al (2011) Intensive glycaemic control for patients with type 2 diabetes: systematic review with meta-analysis and trial sequential analysis of randomised clinical trials. BMJ 343:d6898

11. Yudkin JS, Lipska KJ (2012) Hard end points are needed for intensive glycaemic control in patients with type 2 diabetes. BMJ 344:e708

12. Blood Pressure Lowering Treatment Trialists' Collaboration (2005) Effects of different blood pressure-lowering regimens on major cardiovascular events in individuals with and without diabetes mellitus. Arch Intern Med 165:1410-1419

13. The ACCORD Study Group (2010) Effects of intensive bloodpressure control in type 2 diabetes mellitus. N Engl J Med $362: 1575-1585$
14. Cholesterol Treatment Trialists' (CTT) Collaborators (2008) Efficacy of cholesterol-lowering therapy in 18686 people with diabetes in 14 randomised trials of statins: a meta-analysis. Lancet 371:117-125

15. De Berardis G, Sacco M, Strippoli GFM et al (2009) Aspirin for primary prevention of cardiovascular events in people with diabetes: meta-analysis of randomized controlled trials. BMJ 339:b4531

16. Third Report of the Expert Panel on Detection, Evaluation, and Treatment of High Blood Cholesterol in Adults (Adult Treatment Panel III) Calculator. Available from http://hp2010.nhlbihin.net/ atpiii/calculator.asp. Accessed 26 March 2012

17. The University of Edinburgh Risk Calculator. Available from http://cvrisk.mvm.ed.ac.uk/calculator/calc.asp. Accessed 26 March 2012

18. NHS Evidence (2012) Clinical knowledge summaries. Available from www.cks.nhs.uk/cvd_risk_assessment_and_management/ management/detailed_answers/assessment_of_cvd_risk/ cvd_risk_calculators\#. Accessed 26 March 2012

19. Griffin SG, Borch-Johnsen K, Davies MJ et al (2011) Effect of early intensive multifactorial therapy on 5-year cardiovascular outcomes in individuals with type 2 diabetes detected by screening (ADDITION-Europe): a cluster-randomised trial. Lancet 378:156167

20. Rahman M, Simmons RK, Hennings SH, Wareham NJ, Griffin SJ (2012) How much does screening bring forward the diagnosis of type 2 diabetes and reduce complications? Twelve year follow-up of the Ely cohort. Diabetologia. doi:10.1007/s00125-011-2441-9

21. Simmons RK, Rahman M, Jakes RW et al (2011) Effect of population screening for type 2 diabetes on mortality: long term followup of the Ely cohort. Diabetologia 54:312-319

22. Adriaanse MC, Snoek FJ (2006) The psychological impact of screening for type 2 diabetes. Diabetes Metab Res Rev 22:20-25

23. Adriaanse MC, Snoek FJ, Dekker JM et al (2004) No substantial psychological impact of the diagnosis of type 2 diabetes following targeted population screening: the Hoorn screening study. Diabet Med 21:992-998

24. Park P, Simmons RK, Prevost AT, Griffin SJ (2008) Screening for type 2 diabetes is feasible, acceptable, but associated with increased short-term anxiety: a randomized controlled trial in British general practice. BioMed Public Health 8:350

25. Eborall HC, Griffin SJ, Prevost T, Kinmonth AL, French DP, Sutton S (2007) Psychological impact of screening for type 2 diabetes: controlled trial and comparative study embedded in the ADDITION (Cambridge) randomised controlled trial. BMJ 335:486

26. Paddison CAM, Eborall HC, Sutton S et al (2009) Are people with negative diabetes screening tests falsely reassured? Parallel group cohort study embedded in the ADDITION (Cambridge) randomised controlled trial. BMJ 339:b4535

27. Janssen PGH, Gorter KJ, Stolk RP, Akarsubasi M, Rutten GEHM (2008) Three years follow-up of screen-detected diabetic and nondiabetic subjects: who is better off? The ADDITION Netherlands study. BMC Family Practice 9:67

28. McCabe CJ, Stevenson RC, Dolan AM (1998) Evaluation of a diabetic foot screening and protection programme. Diabet Med $15: 80-84$ 